Case Report

\title{
Atypical Haemolytic Uraemic Syndrome Associated with Clostridium difficile Infection Successfully Treated with Eculizumab
}

\author{
Joshua M. Inglis $\mathbb{D}^{1},{ }^{1}$ Jeffrey A. Barbara, ${ }^{2,3}$ Rajiv Juneja, ${ }^{2,3}$ Caroline Milton, ${ }^{2,3}$ \\ George Passaris, ${ }^{2}$ and Jordan Y. Z. Li (iD) ${ }^{2,3}$ \\ ${ }^{1}$ Department of General Medicine, Royal Adelaide Hospital, Adelaide, SA, Australia \\ ${ }^{2}$ Department of Renal Medicine, Flinders Medical Centre, Adelaide, SA, Australia \\ ${ }^{3}$ School of Medicine, Flinders University, Adelaide, SA, Australia \\ Correspondence should be addressed to Jordan Y. Z. Li; Jordan.Li@sa.gov.au
}

Received 14 December 2017; Revised 12 March 2018; Accepted 4 April 2018; Published 10 May 2018

Academic Editor: Yoshihide Fujigaki

Copyright ( 2018 Joshua M. Inglis et al. This is an open access article distributed under the Creative Commons Attribution License, which permits unrestricted use, distribution, and reproduction in any medium, provided the original work is properly cited.

Clostridium difficile infection is a rare precipitant of atypical haemolytic uraemic syndrome (aHUS). A 46-year-old man presented with watery diarrhoea following an ileocaecal resection. He developed an acute kidney injury with anaemia, thrombocytopaenia, raised lactate dehydrogenase, low haptoglobin, and red cell fragments. Stool sample was positive for C. difficile toxin B. He became dialysis-dependent as his renal function continued to worsen despite treatment with empiric antibiotics and plasma exchange. The ADAMTS13 level was normal consistent with a diagnosis of aHUS. The commencement of eculizumab led to the resolution of haemolysis and stabilisation of haemoglobin and platelets with an improvement in renal function.

\section{Background}

Atypical haemolytic uraemic syndrome (aHUS) is a rare disorder characterised by thrombocytopaenia and evidence of microangiopathic haemolysis. ADAMTS13 levels are normal and STEC (Shiga toxin-producing E. coli) is not present in the stool. The pathogenesis involves activation of complement via the alternative pathway leading to a thrombotic microangiopathy with end-organ involvement predominantly affecting the renal and neurological systems [1]. The precipitants for aHUS include complement regulation deficits, infections, drugs, and pregnancy [2]. Clostridium difficile infection has been identified as a rare precipitant [3-7].

\section{Case Report}

A 46-year-old man with no past medical history and no regular medications presented to a rural hospital with an acute abdomen. An explorative laparotomy revealed a small bowel obstruction secondary to a congenital band with an associated strangulated segment that required an ileocaecal resection. The patient received antibiotic cover with cephazolin, gentamicin, and metronidazole in addition to opioid analgesia. On the tenth postoperative day he had watery diarrhoea. On the following day the patient became anuric with moderate hypertension (BP 160/80).

Blood tests revealed an acute kidney injury with a sudden rise in creatinine from 71 to $307 \mu \mathrm{mol} / \mathrm{L}$ (reference range RR 50-120 $\mu \mathrm{mol} / \mathrm{L})$. The blood film showed red cell fragments (18-24 per HPF) together with an acute anaemia, nadir haemoglobin $68 \mathrm{~g} / \mathrm{L}$ (RR 110-150 g/L), and thrombocytopaenia, nadir platelet count $87 \times 10^{9} / \mathrm{L}(\mathrm{RR} 150-450 \times$ $\left.10^{9} / \mathrm{L}\right)$. Further blood tests revealed a lactate dehydrogenase (LDH) of 1657 (RR 110-230 U/L), a haptoglobin of 0.09 (RR 0.50-2.50), a bilirubin of 86 (RR 2-24), and a negative direct antibody test (DAT). Complement components were low with C3 of 0.75 (RR 0.85-1.60) and C4 of 0.09 (RR 0.12-0.36). There was a coinciding derangement in the liver function tests with gamma-glutamyl transferase (GGT) 589 IU/L $(\mathrm{RR}<60 \mathrm{IU} / \mathrm{L})$, alkaline phosphatase (ALP) $112 \mathrm{IU} / \mathrm{L}(\mathrm{RR}$ $<55 \mathrm{IU} / \mathrm{L})$, aspartate transaminase (AST) $60 \mathrm{IU} / \mathrm{L}(\mathrm{RR}<$ $55 \mathrm{IU} / \mathrm{L})$, and alanine transaminase (ALT) 209 IU/1 (RR < 


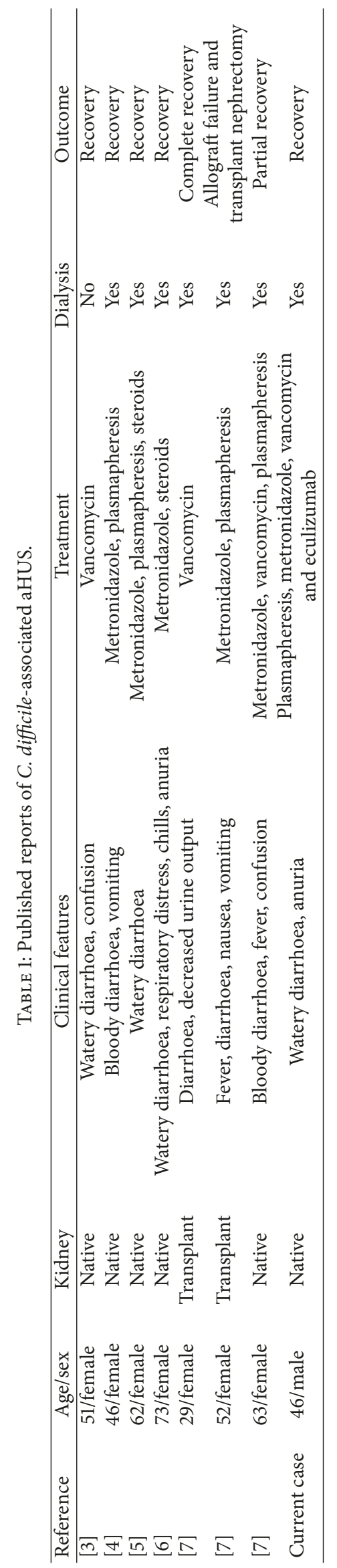


$45 \mathrm{IU} / \mathrm{L})$ which was attributed to critical illness. There was an associated coagulopathy with INR 4.9 (range 0.9-1.2), APTT 67 (range 24-38), fibrinogen 3.0 (range 1.5-4), and D-dimer 3.2 that had likely resulted from the hepatic dysfunction.

The stool sample was positive for Clostridium difficile toxin B and negative for Shiga toxin on two occasions. Stool culture was negative for other enteric pathogens. ADAMTS13 activity was $69 \%$ which is within the normal reference range. Autoimmune screen for ANA, dsDNA, and ANCA was negative. Renal tract ultrasound revealed normal sized kidneys with no hydronephrosis. Liver ultrasound showed hepatomegaly $(18 \mathrm{~cm})$ with no evidence of biliary obstruction. Complement factor $\mathrm{H}(\mathrm{CFH})$ gene analysis did not reveal a mutation.

The patient received daily plasma exchange and haemodialysis in the Intensive Care Unit. Intravenous metronidazole and oral vancomycin were given as antibiotic therapy for C. difficile. Multiple blood transfusions were required to maintain the haemoglobin. The patient required intubation and ventilation for hypoxic respiratory failure for a period of 18 days.

Eculizumab infusions were commenced with the cessation of plasma exchange. The protocol involved infusions of $900 \mathrm{mg}$ weekly for four weeks followed by a single infusion of $1200 \mathrm{mg}$ in the fifth week. During the maintenance phase the patient received fortnightly infusions of $1200 \mathrm{mg}$ for one year.

Eculizumab treatment led to a normalisation in haemoglobin, platelet count, $\mathrm{LDH}$, and haptoglobin over the following fortnight. The patient then became dialysis-independent and serum creatinine stabilised at $200 \mu \mathrm{mol} / \mathrm{L}$ prior to discharge.

He continued to receive eculizumab as an outpatient for one year. There was no relapse of disease on eculizumab cessation. He continues to be monitored with regular blood tests. His renal function remains stable at a new baseline creatinine of $130 \mu \mathrm{mol} / \mathrm{L}$ and there is no evidence of haemolysis.

\section{Discussion}

Atypical haemolytic uraemic syndrome is a clinical diagnosis based on microangiopathic haemolytic anaemia, thrombocytopaenia, and acute kidney injury in the absence of STEC with normal ADAMTS13 activity. Episodes may be precipitated by infection in patients with a genetic susceptibility to complement dysregulation. However, specific complement gene pathway mutations are found in only $50-60 \%$ of cases of true aHUS [8].

This case provides further evidence for $C$. difficile infection as a rare precipitant of aHUS. There are seven reported adult cases of $C$. difficile-associated aHUS with this being the first to occur in an adult male and following abdominal surgery [3-7]. The characteristics of these cases are listed in Table 1. While all cases have shared similar clinical presentations of anaemia, thrombocytopaenia and acute kidney injury, the nature of diarrhoea has varied with both watery and bloody stools being reported. Confusion has been reported in four of eight reported cases suggesting that neurological involvement may occur. However, confusion was not present in our case.
Various treatments have been used in the reported cases of $C$. difficile-associated aHUS including antibiotics, steroids, and plasma exchange with generally favourable outcomes in all but one patient who developed allograft failure and required graft nephrectomy [7]. There have been no documented recurrences of $C$. difficile-associated aHUS.

Terminal complement inhibitors have emerged as an effective therapy for aHUS. Eculizumab has been shown to control haemolysis and lead to improvements in renal function [9]. This is the first reported case of C. difficileassociated aHUS to be treated with eculizumab in addition to conventional therapies. The patient responded favourably with resolution of haemolysis, normalisation of haemoglobin and platelets, and an improvement in renal function. There has been no recurrence of disease two years after ceasing eculizumab. Eculizumab may be an effective agent for achieving resolution of haemolysis and stabilising renal function in patients with $C$. difficile-associated aHUS.

\section{Consent}

The patient has given informed consent for this case report to be published.

\section{Disclosure}

The authors confirm that this case report has not been published previously except in abstract format.

\section{Conflicts of Interest}

The authors declare that they have no conflicts of interest.

\section{References}

[1] D. Kavanagh, S. Raman, and N. S. Sheerin, "Management of hemolytic uremic syndrome," F1000Prime Reports, vol. 6, article no. A119, 2014.

[2] D. Kavanagh, T. H. J. Goodship, and A. Richards, "Atypical haemolytic uraemic syndrome," British Medical Bulletin, vol. 7778, no. 1, pp. 5-22, 2006.

[3] A. Mogyorosi and M. D. Carley, "Hemolytic-uremic syndrome associated with pseudomembranous colitis caused by clostridium difficile," Nephron, vol. 76, no. 4, p. 491, 1997.

[4] C. C. Mbonu, D. L. Davison, K. M. El-Jazzar, and G. L. Simon, "Clostridium difficile Colitis Associated With HemolyticUremic Syndrome," American Journal of Kidney Diseases, vol. 41, no. 5, pp. e14.1-e14.5, 2003.

[5] M. Keshtkar-Jahromi and M. Mohebtash, "Hemolytic uremic syndrome and " Journal of Community Hospital Internal Medicine Perspectives (JCHIMP), vol. 2, no. 3, p. 19064, 2012.

[6] E. Kalmanovich, O. Kriger-Sharabi, E. Shiloah, N. Donin, Z. Fishelson, and M. J. Rapoport, "Atypical hemolytic uremic syndrome associated with Clostridium difficile infection and partial membrane cofactor protein (CD46) deficiency," Israel Medical Association Journal, vol. 14, no. 9, pp. 586-587, 2012.

[7] A. S. Alvarado, S. V. Brodsky, T. Nadasdy, and N. Singh, "Hemolytic uremic syndrome associated with clostridium difficile infection," Clinical Nephrology, vol. 81, no. 4, pp. 302-306, 2014. 
[8] M. Noris and G. Remuzzi, "Atypical hemolytic-uremic syndrome," The New England Journal of Medicine, vol. 361, no. 17, pp. 1676-1687, 2009.

[9] C. M. Legendre, C. Licht, P. Muus et al., "Terminal complement inhibitor eculizumab in atypical hemolytic-uremic syndrome," The New England Journal of Medicine, vol. 368, no. 23, pp. 21692181, 2013. 


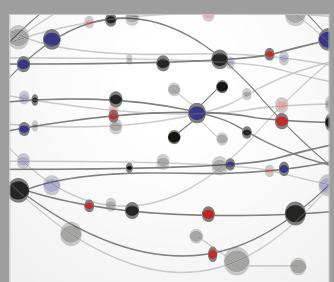

The Scientific World Journal
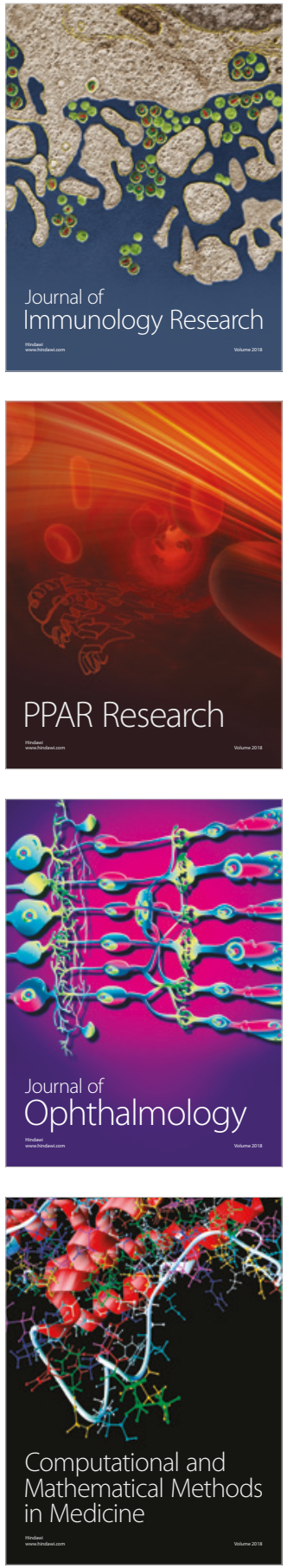

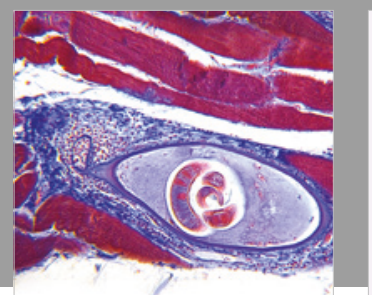

Gastroenterology Research and Practice

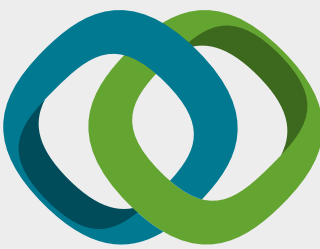

\section{Hindawi}

Submit your manuscripts at

www.hindawi.com
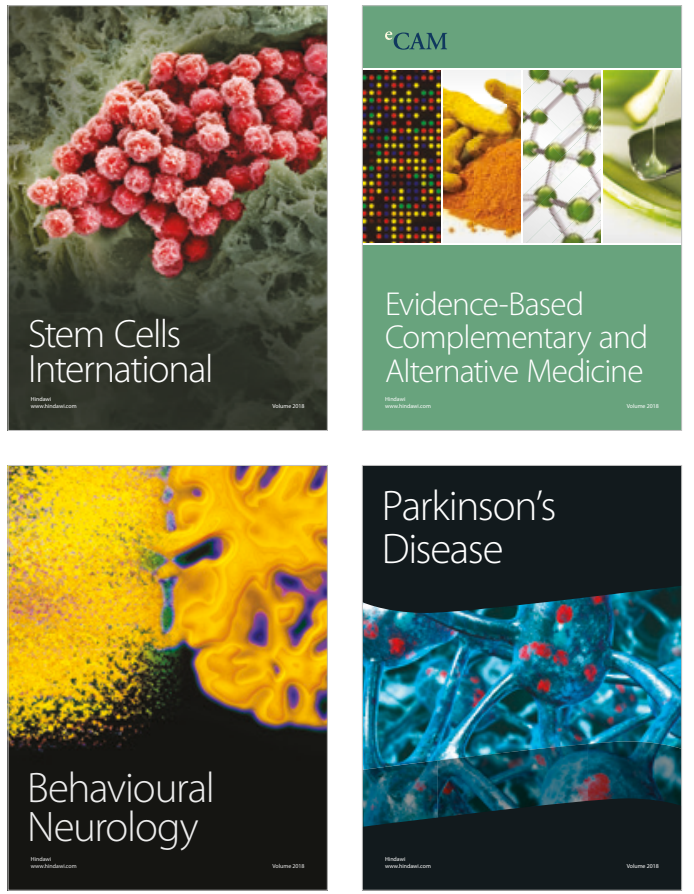

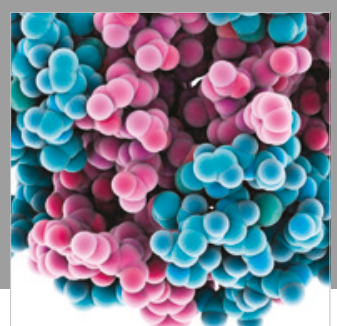

ournal of

Diabetes Research

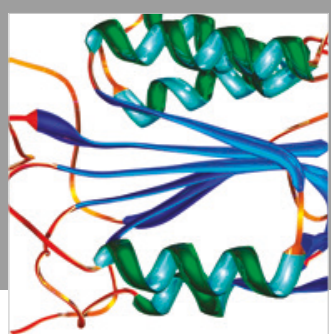

Disease Markers
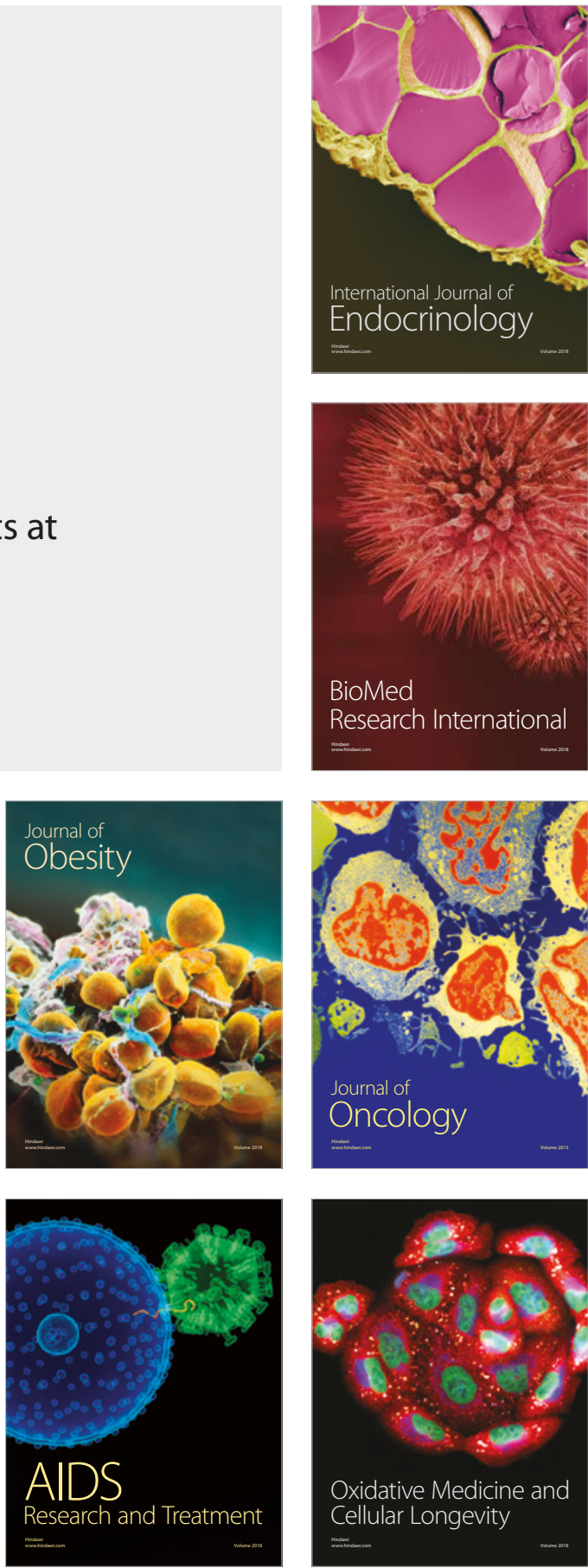